\title{
THE CONTINUOUS MONITORING OF SELECTED RAILWAY STRUCTURES USING THE AUTONOMOUS DATA LOGGER
}

This contribution focuses on issue of the autonomous monitoring of selected dynamic parameters of the railway lines test sections under conditions in the Czech Republic. Description of the possibilities of such data collection, including the designed hardware and software, is a part of this contribution. At the same time, the contribution describes application of such a measuring system, comparing the selected dynamic parameters of the two types of switches, the first one was a switch with a standard fastening node, the other one with an elasticized fastening node. Recommendations and conclusions of railway infrastructure manufacturers and railway line managers are parts of this contribution, as well.

Keywords: data logger, vibrations, monitoring, railway

\section{Introduction}

The railway line (or the tram line) represents a multilayer system determined for the motion of railway vehicles including the equipment necessary for ensuring the safety and smoothness of the railway traffic. The traffic line is formed by the superstructure and the substructure. By the substructure is understood the railway earth bed and the structures that completely or partially substitute protective structures and equipment. Its task is to support the superstructure. The track bed forms the boundary between the substructure and the superstructure [1].

The superstructure is formed by the railway track, which carries and directs vehicles. The superstructure is constructed either as a classic one with the gravel ballast or as a slab track. It should be mentioned that in the Czech Republic the ballast-free track represents the so-called nonconventional structure of the superstructure. Its application occurs especially in special cases like for instance is the tram track, the underground or the track passing through tunnels. The rail of the ballast-free track is mostly fixed to a concrete slab or sleeper, which is fixed to the slab. It should be noted that the text to follow deals with the problems focused on the classic superstructure.

The fundamental part of the superstructure is formed by rails, turnouts, fasteners, sleepers and the railway bed. Rails, together with sleepers, fasteners and the track fastenings, constitute the track length. Its main function is to carry the vehicle and the load, to lead the vehicle geometrically along the trajectory, and by its elasticity to absorb dynamic effects and also to distribute the load into other layers of the superstructure and substructure.

The main function of the gravel ballast is to resist the vertical, cross and longitudinal forces and at the same time to hold the track length in a precise geometrical position. This function is conditioned by suitable properties of gravel grains. Their size must be optimal, the compaction must be sufficient (single grains must be mutually placed so that there may be the smallest possible space between them) and they must be mutually wedged in by their sharp edges [2].

Vibrations of the superstructure are affected by its quality, by operation and structural conditions, climatic phenomena and especially by the dynamic loading by the wheel pairs of the rail vehicles. Related to the rising speed on the railway tracks, the dynamic effects acting on the rail structure increase, as well. These effects negatively influence the rails and lead to the development of failures and defects. Defects usually become evident in the disintegration of the geometric parameters of the rail, which consequently leads to the repeated increase of dynamic effects, which has negative affect on the functionality of the whole system. The result is a malfunction of the entire system and thus also endangering the continuity and safety of the rail traffic. It may be unambiguously said that the solution of the majority of structural problems rests in the right understanding of dynamic effects acting in the railway track structure.

The basic claim put on single parts of the railway tract is their functional reliability together with the safety of the railway operation. Although the superstructure has been practically developed to its perfection during more than 150-year railways, new technical solutions can still be found. This holds good especially for different types of turnout structures, various rail fastenings, various types of rail pads, sleepers, anti-vibration measures etc. It is suitable to say that each part of the rail structure is exposed to considerable static and dynamic stresses.

Dusan Janostik ${ }^{1, *}$, Viktor Nohal ${ }^{1}$, Herbert Seelmann ${ }^{2}$, Jaroslav Smutny $^{1}$

${ }^{1}$ Institute of Railway Structures and Constructions, Faculty of Civil Engineering, Brno University of Technology, Czech Republic

${ }^{2}$ Rail Data Services Austria GmbH \& Co KG, Wien, Austria

*E-mail of corresponding author: janostik.d@fce.vutbr.cz 


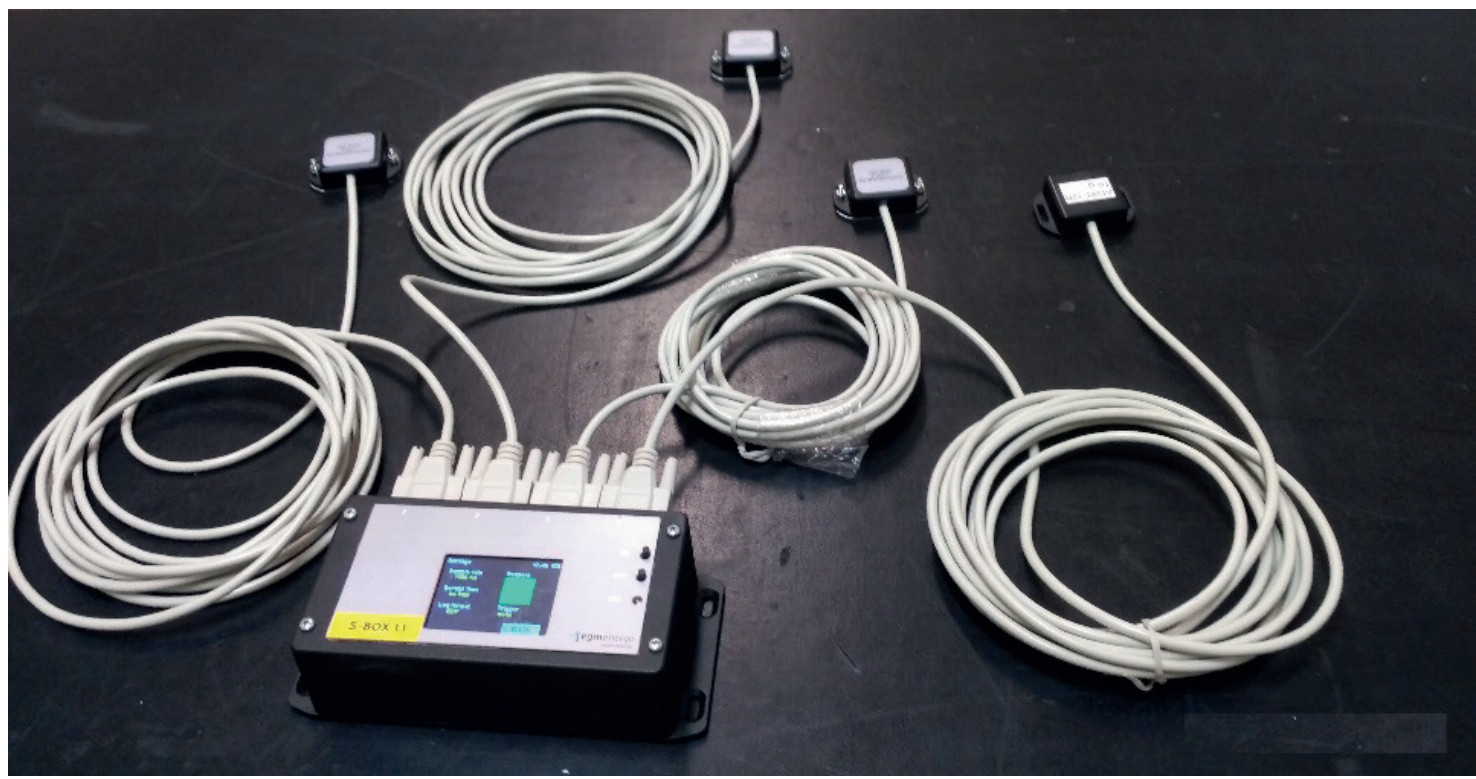

Figure 1 The data logger set with acceleration sensors

Permanent pressure on increasing the transport speed and the operational load of tracks causes a magnificent development of new technologies in the railway traffic. Concerning the modernization of railway lines, this qualitative shift also refers to the Czech and Slovak railway tracks [3]. Decision on modernization of the lines has become an impulse to develop all the branches of the railway traffic both in the field of vehicles and in the field of the infrastructure. Development and application of new experimental procedures for evaluation of the quality and usefulness of particular structural solutions must be in accordance with this trend.

\section{The measurement technology}

Measurements performed on real railway structures are very important. Field measurements take account of the stochastic character of effect of the train sets and properties of the railway line construction. For that purpose, the so-called test sections have been established in the railway network in the Czech Republic to test new structures of the superstructure and the substructure in the long run. Demands put on test sections are usually very strict. This applies primarily to quality of construction and/ or reconstruction, the monitoring of selected parameters, and the access and establishment of measuring points. It should be noted that a new structure to be tested is applied in a track together with its reference, classic variant, i.e. construction without innovation.

It should be mentioned that the short-term measurements have currently prevailed. This means that selected parameters of the railway line have been measured for one or several days. The repeatability of those measurements over a period of one year has been relatively low, with the measurements being usually repeated twice a year. This procedure is appropriate in some cases, while for some structures it would be useful to apply a continuous approach. At the beginning of the Fourth Industrial Revolution, it is certainly worth considering thinking about the implementation of the smart railway structures, which would automatically inform a main system of their status and/or any changes in their status.

To achieve this state, the railways structures (superstructure, points, etc.) have to be provided with an autonomous measuring device that would perform continuous measurements, process the measured data and transfer it to a main evaluation system based on proposed scenarios. Therefore, the authors of this contribution have developed a low-cost automatic measuring system [4]. The system allows not only the "smart" collection of basic data but includes also a set of algorithms enabling information on status of the transport structures and their components to be gathered in the real time and stored for future use as required by an administrator or the design department and/ or for monitoring of an impact on the surroundings. The system has been primarily designed to monitor and evaluate selected dynamic characteristics (vibrations, deformation). The monitoring system provides large data volumes, however, not all the data need to be stored. It is more useful to pre-process data continuously and to store only selected information. The data storage process includes algorithms of intelligent sorting and processing.

\section{Description of the measuring system}

The entire measuring system was designed as a twolevel one. The first level is a data logger, the second is an evaluation software running on a main computer [4].

The data logger (Figures 1 and 2) is designed as a separate measurement point. It may be used throughout the construction of a railway superstructure with the possibility to record up to 12 analogue quantities in the form of an electric voltage of $0 \mathrm{~V}$ to $5 \mathrm{~V}$. It is primarily intended to record vibrations, noise, deformation, displacements and 


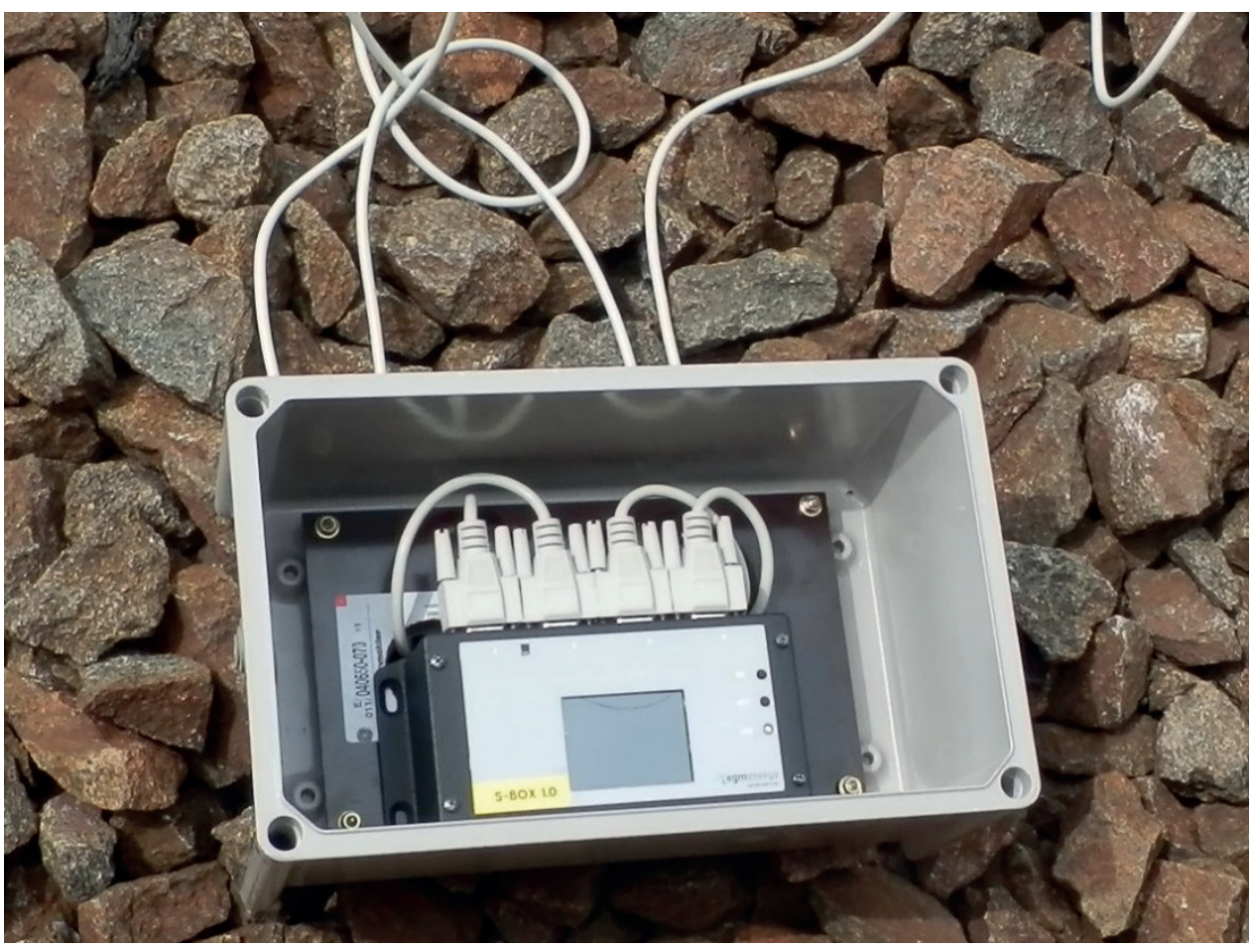

Figure 2 The in-situ view of the data logger

temperature. Quantities are continuously stored on an SD card.

The data logger is built on the microcomputer Teensy platform with a 32-bit ARM Cortex-M4 processor. It can be programmed very similarly to the well-known Arduino system directly via a USB connector. The system is designed to capture a large number of time records of any length at a sampling rate of $100 \mathrm{~Hz}$ to $4000 \mathrm{~Hz}$. This range of sampling frequencies is sufficient to measure vibrations on the track structures. Note that in this area the signals are evaluated up to about $1 \mathrm{kHz}$. The Teensy microcomputer board was completed with additional components for measurement purposes. It was mainly the part forming the measurement chain, power module, data logger synchronization module, data storage to SD card module and a data communication module with a main system. A substantial part of the work was devoted to development of a data logger control system and application software.

The system is equipped with a real-time clock with the possibility to synchronize more of the same data loggers. Measurement and data storage can be done either on external interruptions (change of logic level using, e.g. the gate trigger) or at the set signal level on the selected measurement channel.

After measuring and storing the recording (time events), the system is set to a standby mode to wait for further measurement. The measuring system is equipped with a touchscreen with possibility to set the basic parameters and operations - methods of starting, stopping, setting of measuring time, basic sampling frequency, selection of measuring channels, etc.
The display shows bar charts of the minimum and maximum of all the channels before starting the measurement. The touchscreen makes it possible to select the appropriate number of measuring channels to measure and display averaged maximum and minimum values before measuring to calculate the sensitivity of, for example, the accelerometer sensor. At the same time, it is possible to specify the limit values, whereby the logger reports to the main system a serious problem found in design and sends the data for immediate processing. During the ordinary operation, the main system automatically selects data from individual data loggers for further processing according to the schedule.

The electronics of the data logger is placed in a durable box. The Sub-D9 connectors are used to connect sensors in the proposed version. The system has its battery, as well as the possibility of external charging/powering, for example, by solar panels or by electricity network. The device is designed to be used in real weather conditions. It can be attached to the parts of the railway superstructure (under the rail, on sleepers, etc.).

Several triaxial MEMS-type accelerometer sensors were designed and manufactured for the data logger, two with a measuring range of $200 \mathrm{~g}$ (expected use on the rail), two of $16 \mathrm{~g}$ (assumed use on a sleeper), two with a switchable measuring range of $2 \mathrm{~g} / 6 \mathrm{~g}$ (assumed use outside the track skeleton, possibly in gravel). The MEMS chips from the Analog Devices Company were used to assemble the sensors supplemented with electronics to ensure their power supply and adaptation to the data logger. The produced data logger was tested in the laboratory and field during the year 2019, including the comparative measurements with the Dewetron 2500 system. The 


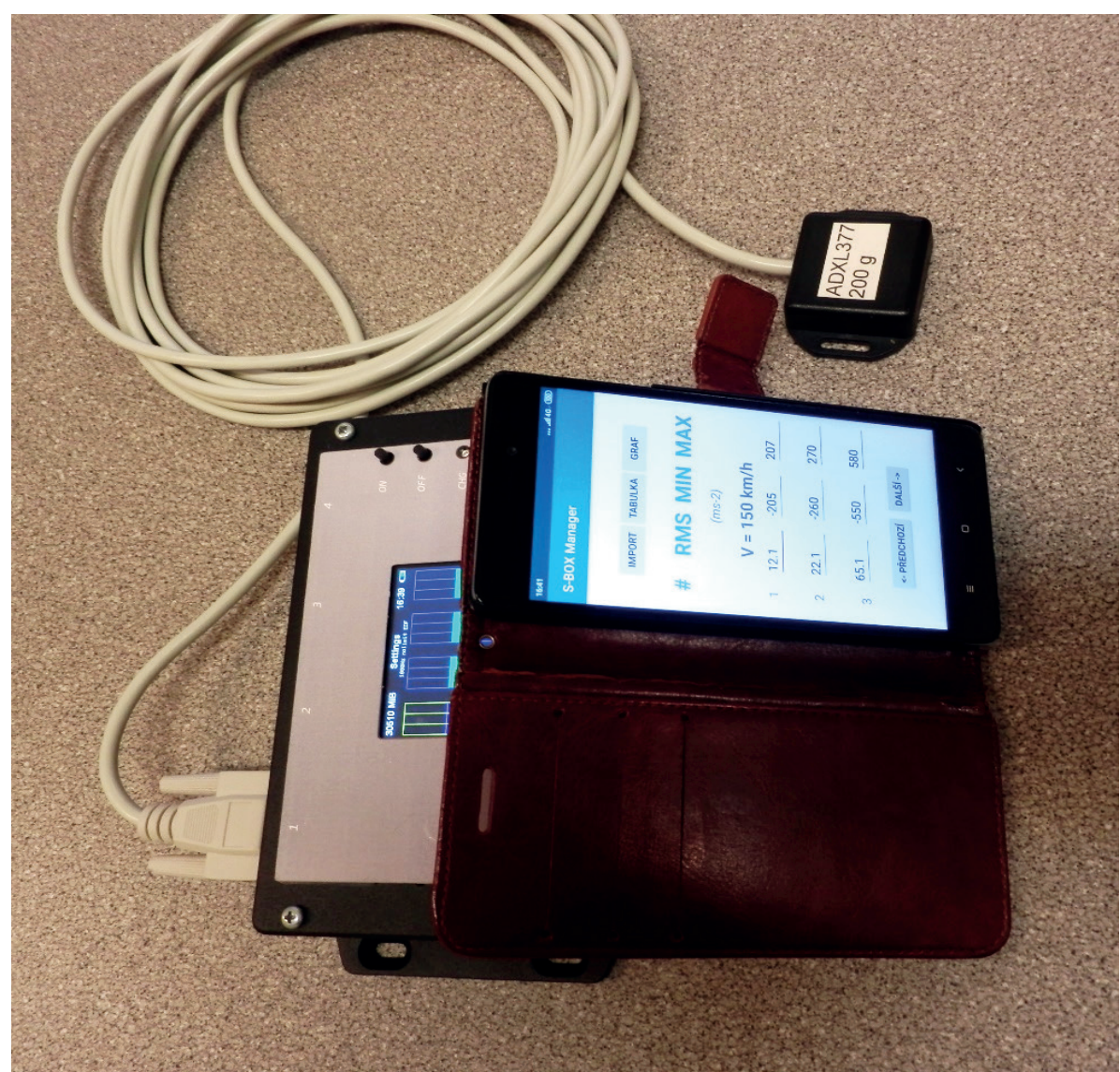

Figure 3 Demonstration of application uploaded to a mobile phone and connection between the mobile phone and a data logger

measuring string was calibrated using a calibration sensor 8305 from Brüel Kjaer and a vibrating calibrator V21D from Metra Mess und Frequenztechnik.

The proposed data logger communicates with a main system in line with current and new trends using WLAN Wi-Fi radio non-cellular technology. The assumed data transmission reaches tens of meters.

The data logger comes with software created to allow the data transfer to the main system and their further data processing. Currently, the software provides the ability to create images with two types of charts. On the left-hand side, there are the time courses of the measured quantity while their amplitude spectra are on the right-hand side. At the same time, other characteristics are identified and counted, e.g. global maxima and minima, RMS values, or floating RMS vector. The software database can be completed with characteristics of locomotives and carriages. By using this data, it is possible to detect the passing train from the measured records. The data read from the data logger, as well as their calculated characteristics, are further stored in created structures of the SQL database. From this database, the pre-processed data can be loaded into the main analysis system. At the end of the data logger description, it is also necessary to mention the possibility to read and analyse the measured data using a mobile phone with the Android operating system. An application was created to read and analyse data over a Wi-Fi connection (Figure 3).

\section{The measurement description}

Monitored switches No. 3 and No. 4 (Figure 4) are situated in the Trebova deviation of the railway station of Usti nad Orlici. Both switches are located on a high embankment. They have the same slenderness (1:12 - 500), both are run by most trains alongside the tip of the frog, and have the same frogs (UIC 60 superstructure, ZPTmonoblock frog). Switch No. 4 has a standard fastening using ribbed plates on concrete sleepers with Skl 24 clamps and it is located on track No. 2. Switch No. 3 has a new UNO3718 fastening using ribbed plates on concrete sleepers with Skl 24 clamps and it is located on track No. 1. The main difference between the above fastenings is mainly in the pad under the foot of rail. Switches are placed on the pre-stressed concrete VPS sleepers.

The dynamic effects were measured after the frog replacement on the new explosion-reinforced manganese frog constructions. It was done both for comparison of both constructions and for verification of measurement methodology based on use of autonomous data logger. The measurement was carried out simultaneously on both switches; the measurement conditions were identical for both measured structures.

The selected design for measuring and investigating the dynamic effects was the switch frog [5]. When a wheel of a train set is passing through, there is a shock and vibration in the frog. The load is distributed through the 


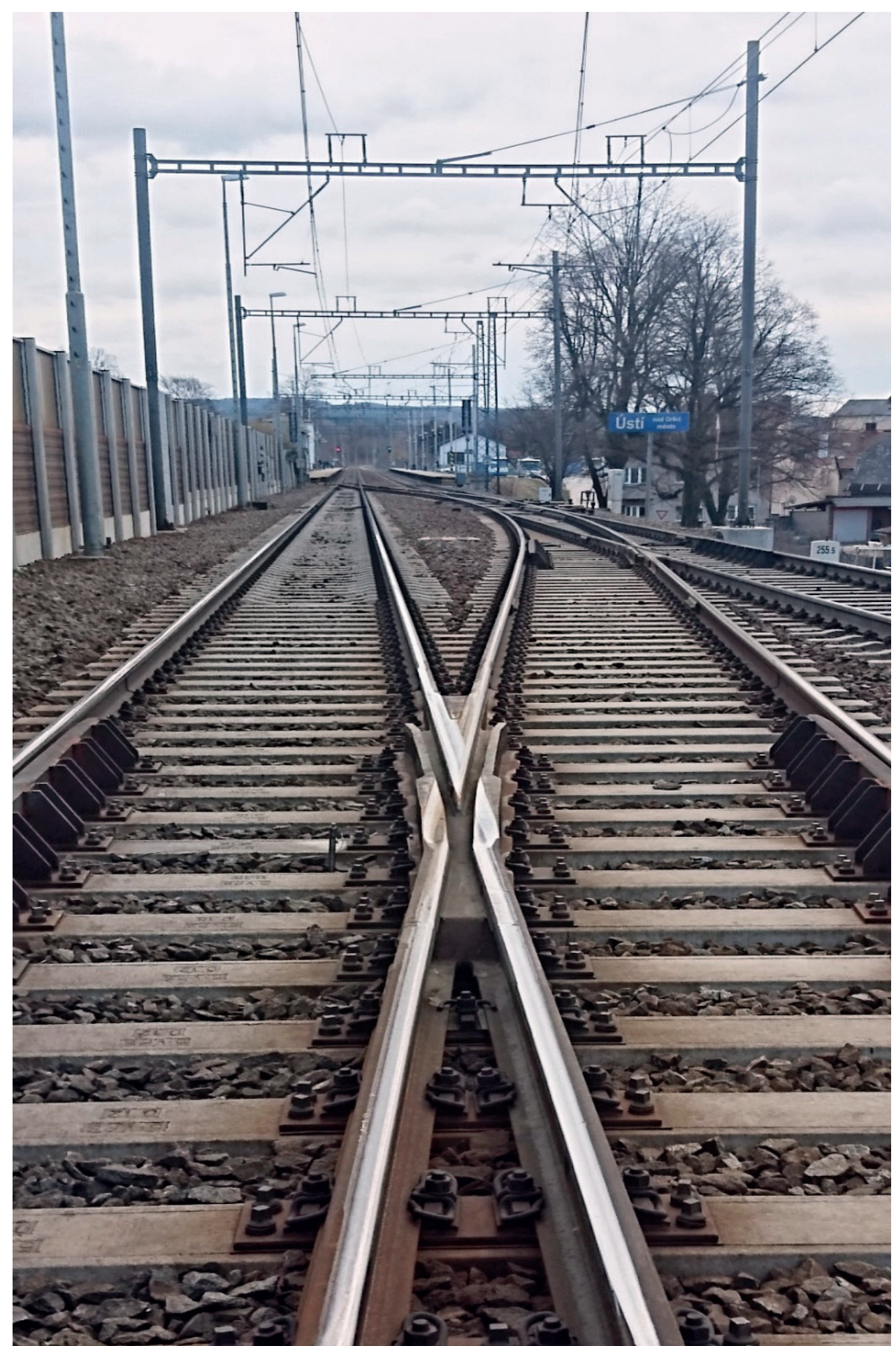

Figure 4 Measurement place, the view of switches No. 3 and No. 4

wheel, into the frog, into the elastic fastening, in which the vibration in the spring washer is partially damped. Part of the vibration spreads and continues through the sleeper to the gravel bed. The energy generated by impact and surface roughness of the wheel-rail surface result in shock and vibrations being passed into the entire superstructure and the ballast bed.

In the rail bed, the gravel grains vibrate, which leads to abrasion. Due to abrasion, the rail bed becomes clogged, the bearing capacity is reduced and the support of the frog structure on the sleeper-gravel bed contact is also worsened. All these can lead to incorrect wheel guidance on the wing rail and can accelerate aging, material degradation in construction and GPK disintegration [6].

Vibrations spread from the source to the structure, in particular in the longitudinal and vertical directions. At the same time, transverse vibrations due to the sinusoidal movement of the wheelset in the rail are also passed into the frog based on the position of the wheel set entrance. Piezoelectric acceleration sensors were chosen to capture 


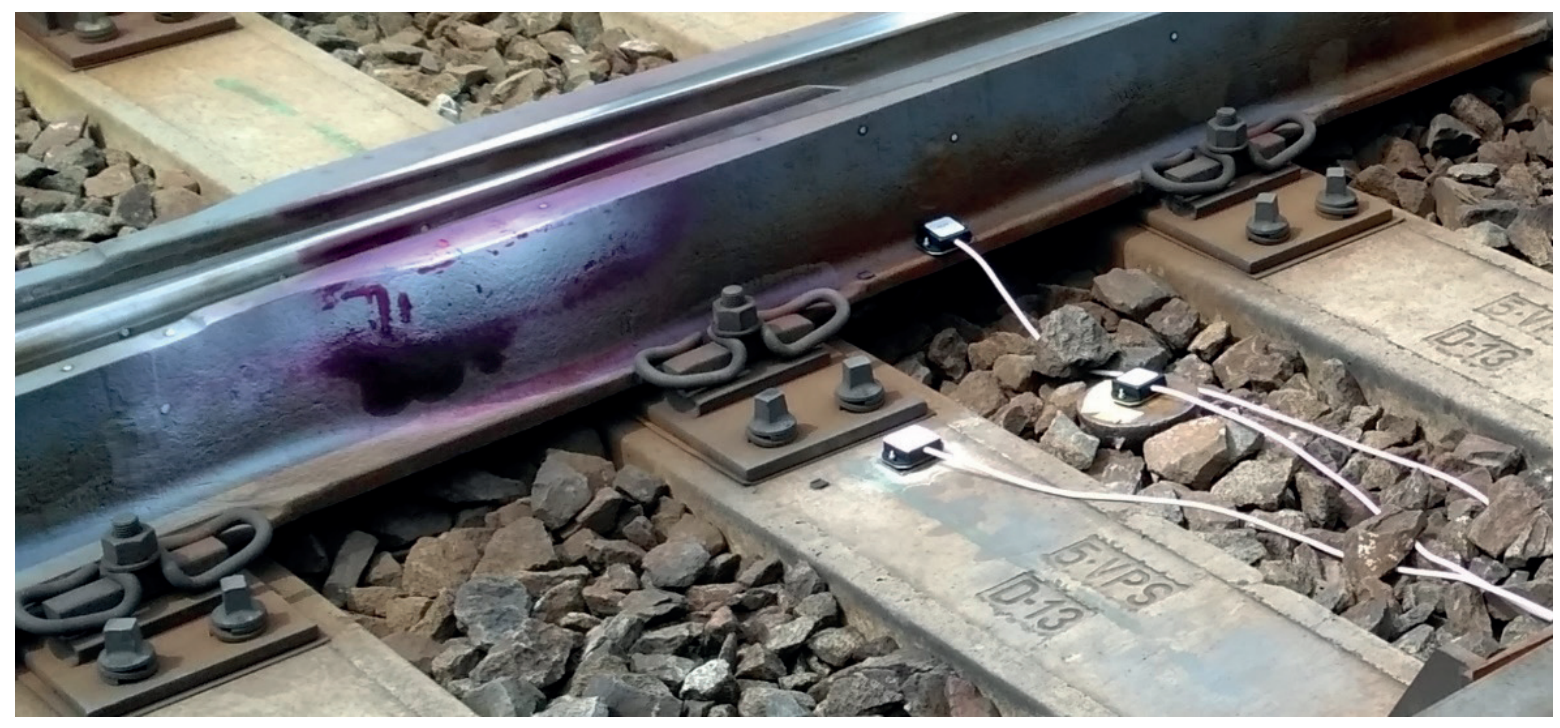

Figure 5 Location of acceleration sensors

the description of vibrations spreading. Piezoelectric sensors belong among the most commonly used sensors for these measurements. Advantages of these sensors are stability wide frequency range and power supply independence. Location of the accelerometers was designed on the base of the wing rail, on the sleeper under the frog tip and in the gravel bed. A three-axis sensor was designed and placed on the heel of the wing rail, which allowed monitoring the dynamic shock caused to the tip of the frog in the vertical direction, transmitted to the foot of the wing rail.

This sensor records vibrations caused by the movement characteristic of the wheelset in the longitudinal and transverse directions. The movement of the wheelset is described as a sinusoid and affects the magnitude of the lateral impact of the phase of movement in which the wheelset enters the frog. The next site under investigation was the transition from the foot of the wing rail to the sleeper under the frog tip. A uniaxial accelerometer sensor was used to detect vibrations on the sleeper. The same was true of monitoring the propagation of vibrations into the gravel bed. To measure the vibrations in the gravel bed, the accelerometer sensor was mounted on a special hemisphere implemented in the gravel layer [7].

The designed data logger was used for measurement of the selected dynamic parameters of both switches for subsequent comparison and evaluation of the effect of application of the spring-loaded fastening in the switch on the vibrations spreading of the switch structures. Note that for this contribution, the transmission of vibrations from the wing rail near the frog tip through the sleeper to the gravel bed was measured (Figure 5). The set of sensors was supplemented with an auxiliary accelerometer, which was placed $2 \mathrm{~m}$ from the frog tip for easy determination of travel speed.

\section{The vibrations assessment methodology}

The test measurement was performed at both positions during the two working days in May 2019 and included train sets in passenger, express and freight train categories. Two developed data loggers were used for measurements. Measurements were performed under the same climatic conditions. The time course of vibration acceleration was the quantity recorded. The start and data storage were accomplished at the pre-set level of a signal detected by the accelerometer placed on a wing rail. Data was transferred to a main computer and evaluated daily. Train sets passing through points were recognized in a main computer by analysing the acceleration measured in a wing rail. A support-vector machine was used to recognize train sets. It is a very promising classification method allowing the train sets to be recognized by an acceleration signal measured in a wing rail by using derived characteristics, such as the floating RMS, the number of local maxima, deviations, etc. Besides, it is also quite convenient to put together a database of locomotives, coaches, and/or whole train sets and their wheel base for the comparison purposes. A classifier had to be set before the measurement itself. The speed of passing train sets was calculated from signals derived from the two accelerometer data recorders (a reference recorder and a recorder placed in a wing rail) and the known distance between them. A method for determining the speed of train sets is based on measuring the time of a wheel passing gradually through two acceleration recorders.

It should be mentioned that vibrations transfer from a wing rail in the vicinity of the crossing frog point through a sleeper to a ballast bed was measured for this contribution. In total, 3 measuring points (Figure 5) were fixed on each switch on which the acceleration sensors (wing rail, sleeper near the fastening and gravel bed near the fastening) were attached. The gravel bed vibrations were measured by an acceleration sensor placed in a special hemisphere pad. This was embedded in the gravel bed in the sleeper crib. Along with the measurement of vibrational characteristics, 
Table 1 Comparison of the effective acceleration values of both monitored turnout structures

\begin{tabular}{|c|c|c|c|c|c|}
\hline \multirow{2}{*}{ Train } & \multirow{2}{*}{$\begin{array}{c}\text { SWITCH } \\
\text { NUMBER }\end{array}$} & \multirow{2}{*}{$\begin{array}{c}\mathrm{V} \\
{\left[\mathrm{km} \cdot \mathrm{h}^{-1}\right]}\end{array}$} & \multicolumn{3}{|c|}{ EFFECTIVE VALUES OF VIBRATION ACCELERATION $\left[\mathrm{m} \cdot \mathrm{s}^{-2}\right]$} \\
\hline & & & $\mathrm{KK}_{\mathrm{z}}$ & $\mathrm{P}_{\mathrm{z}}$ & $\mathrm{SL}_{\mathrm{z}}$ \\
\hline \multirow{2}{*}{ RAILJET } & 3 & 130 & 52.3 & 8.9 & 3.7 \\
\hline & 4 & 130 & 38.2 & 15.0 & 7.4 \\
\hline \multirow{2}{*}{ PENDOLINO } & 3 & 146 & 64.4 & 9.8 & 2.8 \\
\hline & 4 & 160 & 51.5 & 13.3 & 5.9 \\
\hline \multirow[b]{2}{*}{ LEO EXPRESS } & 3 & 132 & 52.3 & 8.0 & 2.8 \\
\hline & 4 & 130 & 36.8 & 14.1 & 4.8 \\
\hline \multirow[b]{2}{*}{ REGIOJET } & 3 & 131 & 53.9 & 10.7 & 3.7 \\
\hline & 4 & 127 & 43.4 & 16.6 & 6.3 \\
\hline \multirow{2}{*}{ R 361} & 3 & 120 & 53.9 & 9.8 & 2.8 \\
\hline & 4 & 120 & 31.6 & 11.6 & 7.2 \\
\hline
\end{tabular}

KKz wing rail, vertical direction

$\mathrm{Pz} \quad$ sleeper - main branch at frog, vertical direction

SLz gravel ballast, vertical direction

a large number of other parameters were measured. Their comparison and analysis are not parts of this paper.

For evaluating vibrations during the passage of train sets around a stationary measuring station, it is advantageous to use the so-called effective value of acceleration $a_{e f}$ or the effective value of acceleration value $L_{\text {aef }}$. This quantity best describes the vibrational behaviour of the passing train. The spreading of vibrational waves from moving trains is characterized by the dependence of the values of the vibration acceleration on the frequency. Therefore, the frequency analysis was also used in the evaluation.

After analysis of the issue, monitoring measurements and calculations, the following methods and parameters were used to analyse the measured data:

- Time waveform of acceleration of the vibration

- Levels of the effective acceleration value

- Frequency analysis using the amplitude spectrum of the linear axis

The mathematical definitions of the individual parameters used to compare the two constructions are briefly summarized. The effective acceleration value is defined by the equation [8]

$a_{e f}=\sqrt{\frac{1}{T} \cdot \int_{0}^{T} a^{2}(t) \cdot d t}\left[\mathrm{~m} \cdot \mathrm{s}^{-2}\right]$,

where $a(t)$ is the instantaneous acceleration value and $T$ is the time for which the effective acceleration value, i.e. the passage time of the train set before the tested construction, is to be determined.

The acceleration frequency spectra were calculated by applying the Fourier transform according to the equation [8-9]:
$A S_{a}(f)=\int_{-\infty}^{\infty} a(t) \cdot e^{-j 2 \pi f t} \cdot d t\left[\mathrm{~m} \cdot \mathrm{s}^{-2}\right]$,

where $f$ is the frequency, $t$ time, and $a(t)$ the acceleration course in the time domain and $A S(f)$ its representation in the frequency domain, $j$ is the imaginary unit.

\section{The measurement assessment}

From the measured records, the basic vibration characteristics, i.e. the effective value of the acceleration value $a_{e f}$, were calculated for each switch and each position of the acceleration sensor, and amplitude spectra for each category of the train. From the calculated data of the effective vertical acceleration value and the time course of acceleration, a table was created for both investigated structures and basic graphs and these were compared. Note that the calculated values given in the table were averaged for each category of the train. The comparison of $L_{\text {aef }}$ values for both measured structures is presented in Table 1. Table 1 shows the following:

The RailJet set has a higher effective vertical acceleration value in the vertical direction for the frog of the switch No. 3. The expected difference is in the effective values on the sleepers, the vibration dampening is higher on the frog at the switch No. 3. The values in the gravel bed are slightly lower for the switch No. 3.

For Pendolino, the effective vertical acceleration values are, according to expectations, the greatest compared to the other kits. Despite different speeds, the acceleration values of the individual sensors are in a similar ratio to the 
Switch No 3, Rail Jet set, $130 \mathrm{~km} / \mathrm{h}$
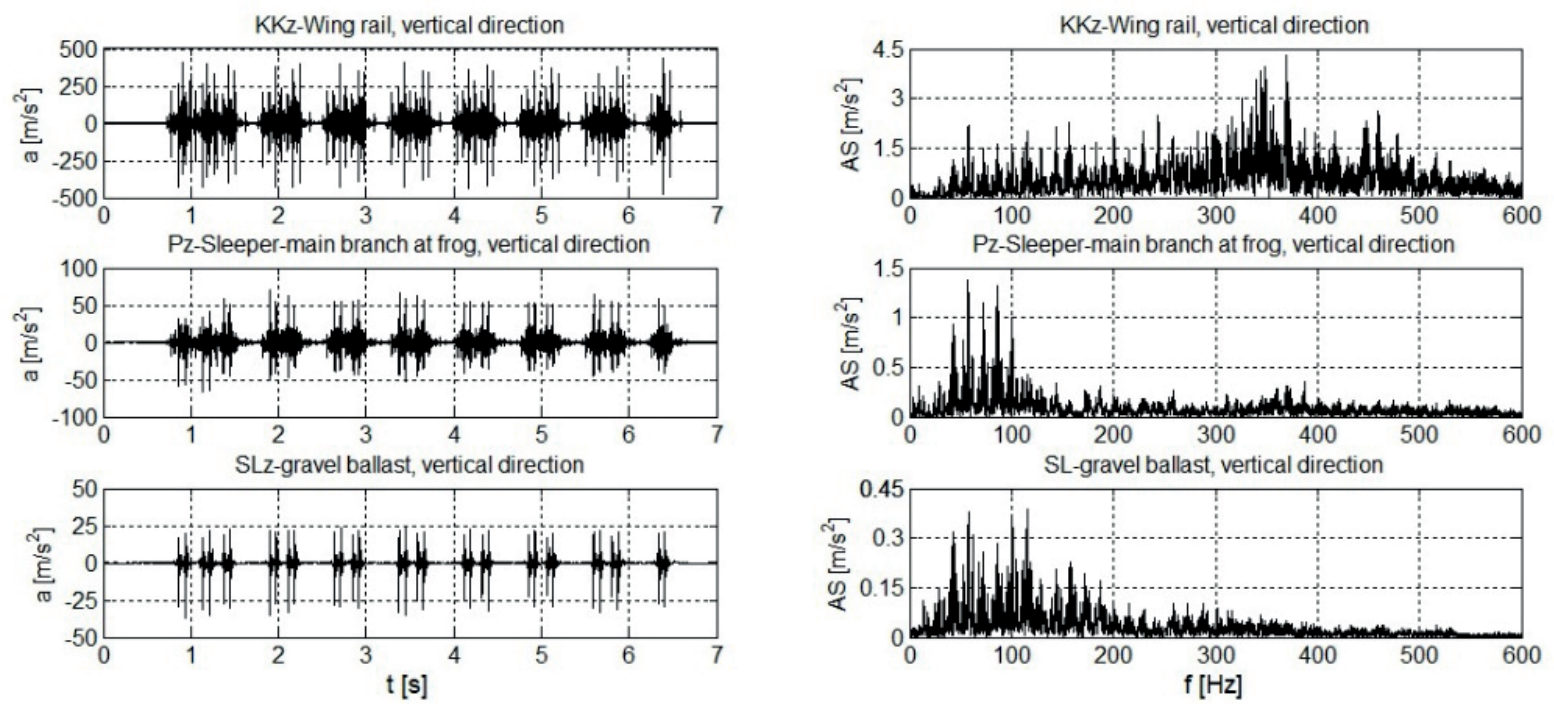

Figure 6 Time and frequency characteristics of switch No. 3, RailJet, speed $130 \mathrm{~km} \cdot \mathrm{h}^{-1}$

Switch No 4, Rail Jet set, 130 km/h
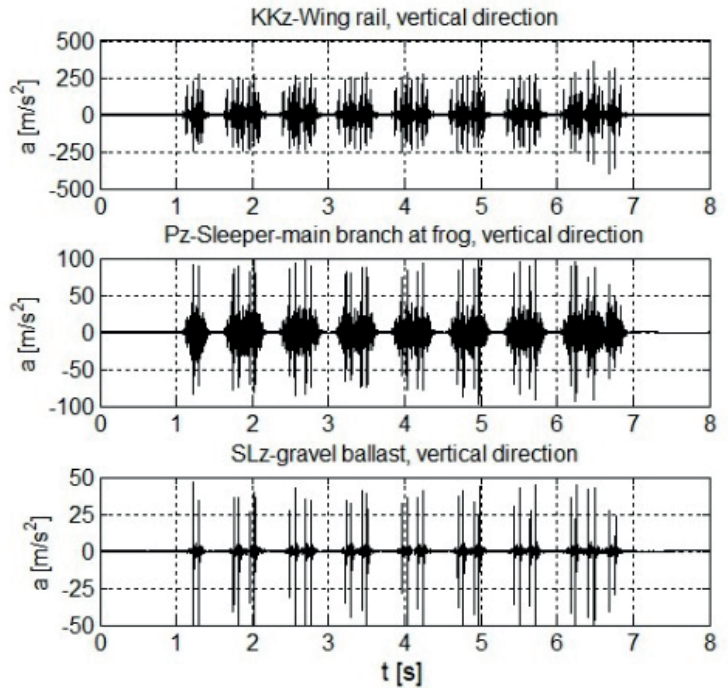
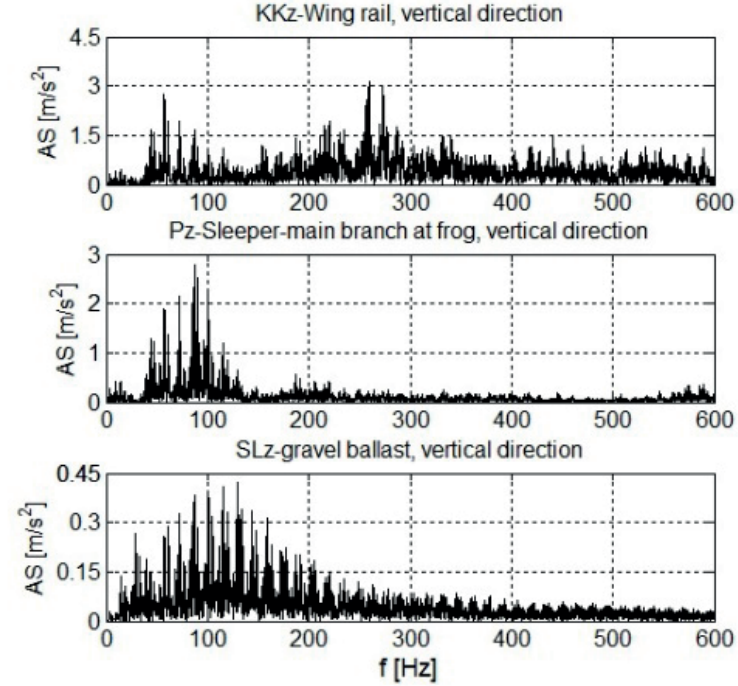

Figure 7 Time and frequency characteristics of switch No. 4, RailJet, speed $130 \mathrm{~km} \cdot \mathrm{h}^{-1}$

RailJet. The same finding applies to Leo Express, RegioJet and 361 series locomotive sets.

In addition, in this case it is important that values in the rail bed are lower for the switch No. 3, i.e. the switch with a sprung fastening against the switch No. 4, i.e. with the classical fastening construction. Note that the attenuation in the track bed is the lowest when the RegioJet was passing through.

Table 1 shows that there is a positive effect of the sprung fastening in the switch No. 3. Due to its use, there is a higher attenuation of vibrations passing from the wing rail to the gravel bed. Analysis and comparison of time records and their amplitude frequency spectra also show similar conclusions. This is evident from the graphs in Figures 6 and 7. The graphs in Figure 6 show the time and frequency characteristics of a switch with a classic mounting node and the graphs in Figure 7 show the outputs of a switch with a flexed mounting node when a high-speed train travels at a speed of $130 \mathrm{~km} \cdot \mathrm{h}^{-1}$. Note that the time and frequency characteristics of vibration measurements are represented by six graphs in both figures. The graphs on the left represent the measured waveforms of the vibrations acceleration at each acceleration sensor. The graphs on the right represent the calculated amplitude frequency spectra.

These graphs show several differences between the two structures. The acceleration values on the wing rail are higher in the switch No. 3 compared to the switch No. 4. The same applies to the amplitude spectra. Only for switch No. 3, maximum spectrum values occur between $300 \mathrm{~Hz}$ and $400 \mathrm{~Hz}$ compared to Switch No. 4, where peaks are achieved at two frequency intervals $(60 \mathrm{~Hz}$ to $70 \mathrm{~Hz}, 200 \mathrm{~Hz}$ to $300 \mathrm{~Hz}$ ). On the sleeper, the lower values were obtained 
in the switch No. 3 compared to the switch No. 4 . This also applies to the frequency spectra. The significant frequency range here is from about $40 \mathrm{~Hz}$ to $120 \mathrm{~Hz}$.

Likewise, the lower values of the switch No. 3 in the ballast bed were achieved. This applies to both time and frequency domains.

\section{Conclusions}

A simple diagnostic system developed at the authors' workplace was presented in the paper. It was designed to be used not only to capture basic dynamic data within railway structures. This system can become a basis for a wider implementation of the concept of intelligent sensing of selected physical quantities on railway (switch) structures and their processing in the form of intelligent diagnostics.

In addition, such a system will find use not only in the field of long-term monitoring of test sections. Based on experience with the presented measuring system, the team recommends continuing in its development. Certainly, it would be appropriate to complement this system with additional sensors and transducers. It would be very beneficial to supplement the proposed equipment with the possibility of measuring the travelled load. A very interesting application area would certainly be integration of the designed data logger into the diagnostic system, which would automatically evaluate the state of the switches, including the occurrence of failures and defects.

From the results, it can be stated that evaluation confirmed the elastic behaviour in the frog seating in the switch No. 3. When moving from wing rail to sleeper, the vibration acceleration values exhibit greater damping than the frog classically imposed.

Based on the work, the authors tested whether the proposed methodology, including the equipment used, was satisfactory. It is sufficient to measure such a range. It represents a suitable complementary method of measurement to diagnose and monitoring the behaviour of the structure. At the same time, the team recommends the use of measuring stones for further measurements, which have a more suitable location and measure the dynamic effects under the tip of the frog in the gravel bed. Note that those were also developed at the Institute of Railway Structures [10].

For more accurate evaluation, it is necessary to carry out measurements more often, preferably continuously using measuring devices built into the measured sections and structures, or more often so that statistical analysis can be included in evaluation. A very interesting idea could be the installation of sensors in the production of switch sleepers located under the frog, where the sensor would measure directly on the contact surface of the sleeper and gravel.

\section{Acknowledgments}

This paper has been supported by the research project The "Switch and Crossing Optimal Design and Evaluation" (S-CODE), supported by the Shift2Rail joint undertaking as a part of the Horizon2020 European research program, focuses on the technological vision for the future generations of switches, under grant agreement No 730849 .

\section{References}

[1] VEIT, P., MARSCHNIG, S. Towards a more sustainable track. Railway Gazette International. 2011, January, p. $42-44$. ISSN 0373-5346.

[2] AUER, F. The influence of elastic components on the track behaviour. OVG Tagung Salzburg. 2012, 104, p. 53-55.

[3] MORAVCIK, M. Analysis of vehicle bogie effects on track structure-nonstationary analysis of dynamic response. Communications - Scientific Letters of the University of Zilina [online]. 2011, 13(3), p. 33-40. ISSN 1335-4205, eISSN 2585-7878. Available from: http://komunikacie.uniza.sk/index.php/communications/article/view/860

[4] SMUTNY, J., PAZDERA, L., JANOSTIK, D., NOHAL, V. The monitoring of railway structures using autonomous datalogger. Akustika. 2019, 33(2), p. 81-93. ISSN 1801-9064.

[5] SMUTNY J., PAZDERA L., NOHAL V., VUKUSICOVA D. Analysis of vibrations on selected structures of railways. Akustika. 2018, 30(2), p. 74-83. ISSN 1801-9064.

[6] SMUTNY, J., PAZDERA, L., NOHAL, V. The analysis of vibrations on selected structures of railways. Akustika. 2019, 31(1), p. 126-135. ISSN 1801-9064.

[7] SMUTNY, J., NOHAL, V. The vibration analysis in the gravel ballast by measuring stone method. Akustika. 2016, 25(1), p. 22-28. ISSN 1801-9064.

[8] SMUTNY, J., PAZDERA, L. The experimental analysis of dynamic processes related to railway transport. Brno: CERM, 2012. ISBN 978-80-7204-827-4.

[9] PAZDERA, L., SMUTNY, J. Evaluation of the railway substructure in the turnout with the movable frog by help of decomposition. In: Applied Methods of the Analysis of Static and Dynamic Loads of Structures and Machines II. Switzerland: Trans Tech Publications, 2016. ISSN 1660-9336, ISBN 978-3-03835-531-1.

[10] Evaluation of dynamic effects acting on switches. Certified methodology, 124/2014-710-VV/1, 2014. 